\title{
Toward Resources and Processes Sustainability: Part II
}

\author{
XIAOFEI GUAN $\mathbb{1}^{1,2}$ \\ 1.-School of Physical Science and Technology, ShanghaiTech University, Shanghai 201210, \\ China. 2.-e-mail: guanxf@shanghaitech.edu.cn
}

Sustainability is playing an increasingly important role in driving innovation. Because of the overwhelming response to this theme, this special topic presents a continuation of February's theme of resources and processes sustainability. In this issue, readers will find articles from various application areas covering structural metal recovery, nonmetallic inclusions in steel, silicon purification, copper slag utilization, and aluminum alloy recycling.

Metals and alloys are widely used for structural applications. Due to degradation, these structural components can gradually become deficient during service and, in some cases, may require expensive and time-consuming replacement to avoid catastrophic failures. In the first paper, Smith and Sampath provide a timely scientific review on spray-clad remanufacturing as an alternative strategy to recover the degraded structural metals. The authors review the current state of the spray-clad remanufacturing technologies and also provide a systematic discussion evaluating the capabilities of the remanufactured systems. Spray-clad remanufacturing shows promise especially for recovering degraded structural metals in place in a convenient and cost-effective way.

High heat input during welding can affect the mechanical properties of steel adversely, especially in heat-affected zones. Utilization of non-metallic inclusions can mitigate this effect and thereby enhance the steel performance and reduce resource waste. In their paper, Wang, Zou et al. apply confocal scanning laser microscopy to perform insitu observation of the inclusions in a shipbuilding steel during heating at $1473 \mathrm{~K}$. Their systematic investigation advances the understanding of inclusion evolution behaviors.

Xiaofei Guan is the JOM advisor for the Recycling and Environmental Technologies Committee of the TMS Light Metals Division and Extraction \& Processing Division, and guest editor for the topic, Toward Resources and Processes Sustainability: Part II, in this issue.
Another paper discusses hydrometallurgical purification of metallurgical-grade silicon using $\mathrm{HCl}-\mathrm{HF}-\mathrm{CH}_{3} \mathrm{COOH}$ leaching, as presented by Tian et al. The addition of $\mathrm{CH}_{3} \mathrm{COOH}$ to $\mathrm{HCl}-\mathrm{HF}$ is reported to enhance the removal of impurities and shorten the required leaching time.

This special topic also features two papers on comprehensive utilization of copper slag. Copper slag is a major byproduct from the copper smelting industry. Typically, it is either disposed of as waste or utilized for blast-cleaning or concrete production. In comparison, recovery of metals from the copper slag can offer both economic and environmental benefits. In this special topic, Guo et al. report on copper recovery in the flotation of copper slag that was modified by adding composite additives. The modification leads to an increase in the copper recovery as well as the copper grade. Another paper focuses on fayalite $\left(\mathrm{Fe}_{2} \mathrm{SiO}_{4}\right)$, the major phase of iron in copper slag. Wang, Peng et al. have investigated the formation mechanism of fayalite by solid-state reaction in the sintering process. This work leads to an improved mechanistic understanding of the fayalite phase formation and could assist the process control in recovering value from fayalite.

The last paper is focused on aluminum-cerium alloy recycling. The successful adoption of a new aluminum alloy in today's automobile industry requires not only technologic innovations but also valid economic analysis of all the stages of the product's life, especially recycling. In this paper, Iyer et al. provide a comprehensive analysis of the recycling supply chain of aluminum-cerium alloy, which provides a guideline for the adoption of new aluminum alloys.

The following papers are published under the topic "Toward Resources and Processes Sustainability: Part II" in the April 2018 issue (vol. 70, no. 4) of $J O M$ and can be accessed via the $J O M$ page at http://link.springer.com/journal/11837/70/4/page/1.

- "Sustainability of Metal Structures via SprayClad Remanufacturing” by Gregory M. Smith and Sanjay Sampath. 
- "Evolution of Inclusions during $1473 \mathrm{~K}$ Heating Process in EH36 Shipbuilding Steel with Mg Addition" by Qiyu Wang, Xiaodong Zou, Hiroyuki Matsuura, and Cong Wang.

- "Effect of $\mathrm{CH}_{3} \mathrm{COOH}$ on Hydrometallurgical Purification of Metallurgical-Grade Silicon Using HCl-HF Leaching" by Chunjin Tian, Haifei Lu, Kuixian Wei, Wenhui Ma, Keqiang Xie, Jijun Wu, Yun Lei, Bin Yang, and Kazuki Morita.

- "Industrial Tests to Modify Molten Copper Slag for Improvement of Copper Recovery" by Zheng- qi Guo, Deqing Zhu, Jian Pan, Feng Zhang, and Congcong Yang.

- "Study on Formation Mechanism of Fayalite $\left(\mathrm{Fe}_{2} \mathrm{~S}\right.$ $\mathrm{iO}_{4}$ ) by Solid State Reaction in Sintering Process" by Zhongbing Wang, Bing Peng, Lifeng Zhang, Zongwen Zhao, Degang Liu, Ning Peng, Dawei Wang, Yinghe He, Yanjie Liang, and Hui Liu.

- "An Economic Model and Experiments to Understand Aluminum Cerium Alloy Recycling" by Ananth V. Iyer, Heejong Lim, Orlando Rios, Zachary Sims, and David Weiss. 